INPLASY

PROTOCOL

To cite: Yang et al. Efficacy and safety of glucocorticoids in the treatment of patients with liver failure: a meta-analysis of randomized controlled trials. Inplasy protocol 202170083. doi:

10.37766/inplasy2021.7.0083

Received: 26 July 2021

Published: 26 July 2021

Corresponding author:

Xiaojie Huang

huangxiaojie78@ccmu.edu.cn

Author Affiliation:

Capital Medical University,

Beijing Youan Hospital.

Support: No.

Review Stage at time of this submission: Preliminary

searches.

Conflicts of interest:

None declared.

\section{Efficacy and safety of glucocorticoids in the treatment of patients with liver failure: a meta-analysis of randomized controlled trials}

Yang, J1; Hu, X2; Wang, X3; Ning, Q4; Gou, C5; Wang, X6; Li, L7; Zhang, L8; Hu, Z9; Huang, $X^{10}$.

Review question / Objective: The differences in the effectiveness and safety of glucocorticoids in the treatment of patients with liver failure.

Condition being studied: Glucocorticoid therapy has been reported to prevent the necrosis of liver cells and provide the possibility of liver regeneration. However, glucocorticoids have not been widely used for the treatment of liver failure. In recent years, with the new generation of nucleoside analogs, proton pump inhibitors, and effective infection control measures, the use of glucocorticoids to liver failure has become much safer. However, the efficacy of glucocorticoids remains debatable. To compare the efficacy of glucocorticoids with conventional medication, we carried out a meta-analysis on patients enrolled in randomized trials, comparing the effectiveness of these two treatment strategies.

INPLASY registration number: This protocol was registered with the International Platform of Registered Systematic Review and Meta-Analysis Protocols (INPLASY) on 26 July 2021 and was last updated on 26 July 2021 (registration number INPLASY202170083).

\section{INTRODUCTION}

Review question / Objective: The differences in the effectiveness and safety of glucocorticoids in the treatment of patients with liver failure.
Rationale: Liver failure is usually accompanied by multiple organ failure, rapid progression and a low survival rate. The sole solution that has been proven to be beneficial is liver transplantation, but its application is limited by the rapid disease progression and lack of donors. Beyond 
the development of interferon and nucleoside analogs, which prevent the rapid progression of the disease to hepatic failure, no effective therapies are available for the treatment of patients with severe liver failure. Therefore, finding a safe and effective therapeutic approach to liver failure is urgent.

Condition being studied: Glucocorticoid therapy has been reported to prevent the necrosis of liver cells and provide the possibility of liver regeneration. However, glucocorticoids have not been widely used for the treatment of liver failure. In recent years, with the new generation of nucleoside analogs, proton pump inhibitors, and effective infection control measures, the use of glucocorticoids to liver failure has become much safer. However, the efficacy of glucocorticoids remains debatable. To compare the efficacy of glucocorticoids with conventional medication, we carried out a meta-analysis on patients enrolled in randomized trials, comparing the effectiveness of these two treatment strategies.

\section{METHODS}

Search strategy: \#1"Hepatic Insufficiency"[MeSH Terms]; \#2 "hepatic" [Title/Abstract]; \#3 "Liver"[MeSH Terms]; \#4 "liver"[Title/Abstract]; \#5"Liver"[Title/ Abstract] OR "Liver"[MeSH Terms] OR "hepatic"[Title/Abstract] OR "Hepatic Insufficiency"[MeSH Terms]; \#6 "failure" [Title/Abstract]; \#7 "failure"[Title/Abstract] AND ("Liver"[Title/Abstract] OR "Liver"[MeSH Terms] OR "hepatic"[Title/ Abstract] OR "Hepatic Insufficiency"[MeSH Terms]); \#8 "Liver Failure"[MeSH Terms]; \#9 "liver failure"[Title/Abstract]; \#10 "hepatic failure"[Title/Abstract]; \#11 "Liver Failure"[MeSH Terms] OR "Liver Failure"[Title/Abstract] OR "hepatic failure" [Title/Abstract]; \#12 ("failure"[Title/ Abstract] AND ("Liver"[Title/Abstract] OR "Liver"[MeSH Terms] OR "hepatic"[Title/ Abstract] OR "Hepatic Insufficiency"[MeSH Terms])) OR ("Liver Failure"[MeSH Terms] OR "Liver Failure"[Title/Abstract] OR "hepatic failure"[Title/Abstract]); \#13 "Glucocorticoids"[MeSH Terms]; \#14"
Glucocorticoids"[Title/Abstract]; \#15 "Methylprednisolone"[MeSH Terms]; \#16 "Methylprednisolone"[Title/Abstract]; \#17 "Dexamethasone"[Title/Abstract]; \#18 "Dexamethasone"[MeSH Terms]; \#19 "hydrocortison"[Title/Abstract]; \#20 "budesonid"[Title/Abstract]; \#21 "methylpredniso*"[Title/Abstract]; \#22 "cortico*"[Title/Abstract]; \#23 "glucocortico*"[Title/Abstract]; \#24"Glucocorticoids"[MeSH Terms] OR "Glucocorticoids"[Title/Abstract] OR "Methylprednisolone"[MeSH Terms] OR "Methylprednisolone"[Title/Abstract] OR "Dexamethasone"[Title/Abstract] OR "Dexamethasone"[MeSH Terms] OR "hydrocortison"[Title/Abstract] OR "budesonid"[Title/Abstract] OR "methylpredniso*"[Title/Abstract] OR "cortico*"[Title/Abstract] OR "glucocortico*"[Title/Abstract]; \#25 (("failure"[Title/Abstract] AND ("Liver"[Title/ Abstract] OR "Liver"[MeSH Terms] OR "hepatic"[Title/Abstract] OR "Hepatic Insufficiency"[MeSH Terms])) OR ("Liver Failure"[MeSH Terms] OR "Liver Failure"[Title/Abstract] OR "hepatic failure"[Title/Abstract])) AND ("Glucocorticoids"[MeSH Terms] OR "Glucocorticoids"[Title/Abstract] OR "Methylprednisolone"[MeSH Terms] OR "Methylprednisolone"[Title/Abstract] OR "Dexamethasone"[Title/Abstract] OR "Dexamethasone"[MeSH Terms] OR "hydrocortison"[Title/Abstract] OR "budesonid"[Title/Abstract] OR "methylpredniso*"[Title/Abstract] OR "cortico* "[Title/Abstract] OR "glucocortico*"[Title/Abstract]); \#26 ("randomized controlled trial"[Publication Type] OR "controlled clinical trial"[Publication Type] OR "randomized"[Title/Abstract] OR "placebo"[Title/Abstract] OR "clinical trials as topic"[MeSH Terms] OR "randomly"[Title/Abstract] OR "trial"[Title]) NOT ("animals"[MeSH Terms] NOT " humans" [M eSH Terms ]); \#27 (("randomized controlled trial"[Publication Type] OR "controlled clinical trial"[Publication Type] OR "randomized"[Title/Abstract] OR "placebo"[Title/Abstract] OR "clinical trials as topic"[MeSH Terms] OR 
"randomly"[Title/Abstract] OR "trial"[Title]) NOT ("animals"[MeSH Terms] NOT "humans"[MeSH Terms])) AND ( ( ("failure"[Title/Abstract] AND ("Liver"[Title/Abstract] OR "Liver"[MeSH Terms] OR "hepatic"[Title/Abstract] OR "Hepatic Insufficiency"[MeSH Terms])) OR ("Liver Failure"[MeSH Terms] OR "Liver Failure"[Title/Abstract] OR "hepatic failure"[Title/Abstract])) AND ("Glucocorticoids"[MeSH Terms] OR "Glucocorticoids"[Title/Abstract] OR "Methylprednisolone"[MeSH Terms] OR "Methylprednisolone"[Title/Abstract] OR "Dexamethasone"[Title/Abstract] OR "Dexamethasone"[MeSH Terms] OR "hydrocortison"[Title/Abstract] OR "budesonid"[Title/Abstract] OR "methylpredniso*"[Title/Abstract] OR "cortico* "[Title/Abstract] OR "glucocortico*"[Title/Abstract])).

Participant or population: Patients with liver failure.

Intervention: Patients with liver failure receiving glucocorticoids at a low dose (less than $50 \mathrm{mg}$, qd or $0.5 \mathrm{mg} / \mathrm{kg}$, qd), or a high dose (60mg, qd or $1 \mathrm{mg} / \mathrm{kg}$, qd).

Comparator: General medical treatment.

Study designs to be included: Randomized controlled trials.

Eligibility criteria: Inclusion: patients with a confirmed diagnosis of liver failure. Exclusion: overlap syndrome patients, liver transplantation patients before study.

Information sources: MEDLINE, Embase, and Cochrane Central Register of Controlled Trials (CENTRAL) were retrieved from each creation date to July 11, 2021.

Main outcome(s): The efficiency (biochemical remission rate, even death, etc.).

Additional outcome(s): safety (incidence of adverse effects of glucocorticoids, such as secondary infection, bleeding etc.) of different doses of glucocorticoids.
Quality assessment / Risk of bias analysis: RoB2.

Strategy of data synthesis: Stata.

Subgroup analysis: Revman $\mathbf{5 . 4 .}$

Sensitivity analysis: Revman $\mathbf{5 . 4 .}$

Country(ies) involved: China.

Keywords: glucocorticoids, liver failure, meta-analysis.

Contributions of each author:

Author 1 - Jun Yang.

Email: lyn2020@ccmu.edu.cn

Author 2 - Xing Hu.

Author 3 - Xin Wang.

Email: 13521101275@139.com

Author 4 - Qiqi Ning.

Author 5 - Chunyan Gou.

Email: goucy@vip.sina.com

Author 6 - Xiaojun Wang.

Email: 13718217343@126.com

Author 7 - Li Li.

Email: 78000062@ccmu.edu.cn

Author 8 - Lili Zhang.

Email: 1768683745@qq.com

Author 9 - Zhongjie Hu.

Email: huzhongjie@ccmu.edu.cn

Author 10 - Xiaojie Huang.

Email: huangxiaojie78@ccmu.edu.cn 